\title{
Driving Cycle Equivalence and Transformation
}

Peter Nyberg, Erik Frisk and Lars Nielsen

The self-archived version of this journal article is available at Linköping University Electronic Press:

http:/ / urn.kb.se/ resolve?urn=urn:nbn:se:liu:diva-118104

N.B.: When citing this work, cite the original publication.

Nyberg, P., Frisk, E., Nielsen, L., (2017), Driving Cycle Equivalence and Transformation, IEEE Transactions on Vehicular Technology, 66(3), 1963-1974.

https:// dx.doi.org/10.1109/TVT.2016.2582079

Original publication available at:

https:/ / dx.doi.org/ 10.1109/TVT.2016.2582079

Copyright: Institute of Electrical and Electronics Engineers (IEEE)

http:// www.ieee.org/ index.html

(C) 2016 IEEE. Personal use of this material is permitted. However, permission to reprint/ republish this material for advertising or promotional purposes or for creating new collective works for resale or redistribution to servers or lists, or to reuse any copyrighted component of this work in other works must be obtained from the IEEE. 


\title{
Driving Cycle Equivalence and Transformation
}

\author{
Peter Nyberg, Erik Frisk, and Lars Nielsen
}

\begin{abstract}
There is a current strong trend where driving cycles are used extensively in vehicle design, especially for calibration and tuning of all powertrain systems for control and diagnosis. In such situations it is essential to capture real driving, and therefore using only a few driving cycles would lead to the risk that a test or a design would be tailored to details in a specific driving cycle. Consequently there are now widespread activities using techniques from statistics, big data and mission modeling to address these issues. For all such methods there is an important final step to calibrate a representative cycle to adhere to fair propulsion requirements on the driven wheels over a cycle. For this a general methodology has been developed, applicable to a wide range of problems involving driving cycle transformations. It is based on a definition of equivalence for driving cycles that loosely speaking defines being similar without being the same. Based on this, a set of algorithms are developed to transform a given driving cycle into an equivalent one, or into a cycle with given equivalence measure. The transformations are effectively handled as a nonlinear program that is solved using general purpose optimization techniques. The proposed method is general and a wide range of constraints can be used.
\end{abstract}

Index Terms-mean tractive force, nonlinear programming, numerical optimization, vehicle design, vehicle propulsion.

\section{INTRODUCTION}

A driving cycle is represented by vehicle speed versus time and is supposed to represent typical driving patterns [1][3]. Therefore driving cycles play a fundamental role in the design of vehicles, and if vehicle manufacturers focus only on a single driving cycle, during the development and design of a vehicle, there is a risk that the design is optimized for this specific driving cycle and the result for another driving cycle may be non-robust and sub-optimal [4], [5].

Design for realistic use is a main driving force behind the current development in usage of driving cycles where the ultimate goals are making good cars both for customers and for upcoming RDE (Real Driving Emissions) evaluations. Customers want their cars to perform well in real driving, and PEMS (Portable Emission Measurement System) will be used to compare cars in real driving by different actors in the field. Further, the new RDE test procedure for Europe was voted in May 2015 by the relevant regulatory committee (Technical Committee of Motor Vehicles - TCMV) and will come into force early 2016, which is a strong indication that the legislations will be altered to address the customers' viewpoint and to assess fuel consumption/emissions in real driving conditions.

Manuscript received May 5, 2015; revised February 11 and June 2, 2016. The work was supported in part by the Swedish Hybrid Vehicle Centre and in part by Linnaeus Center CADICS.

The authors are with the Department of Electrical Engineering, Linköping University, Linköping, 58183 Sweden (e-mail: erik.frisk@liu.se).

Color versions of one or more of the figures in this paper are available online at http://ieeexplore.ieee.org.

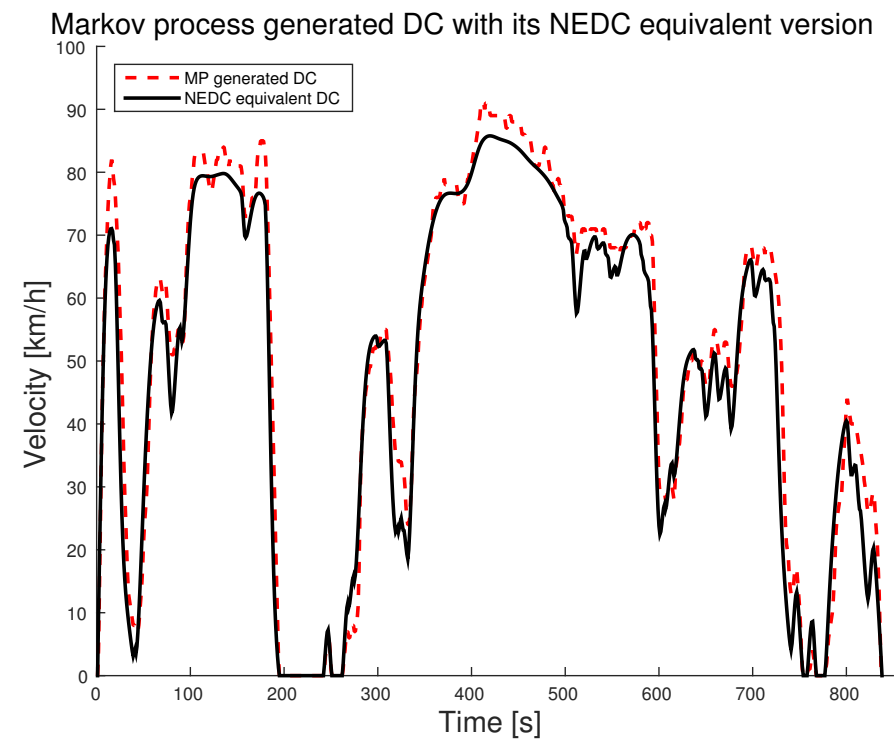

Fig. 1. A generated driving cycle (dashed) using a Markov chain approach and a driving cycle that is equivalent to NEDC (solid).

To be able to meet these requirements, the vehicle development process used in industry must be tailored accordingly. This means that design engineers must have easy and appropriate access to real driving behavior when designing, simulating, and testing their vehicle designs. The only way to have this is to use real driving data and real driving missions. Note that this cannot be achieved by just taking a selection of recorded driving data, since that data may not be representative for the whole ensemble of drives and missions. Neither is it sufficient to use the available homologation test cycles. Consequently there are now widespread activities using techniques from statistics, big data, and mission modeling for finding representative driving cycles, see e.g. [6]. The so generated representative driving cycles differ in propulsion requirement, so to be able to use them in comparative design studies there is an important final step to calibrate a representative cycle to adhere to the propulsion requirements on the driven wheels over a cycle. See for example Fig. 1 where the dashed cycle is a cycle obtained from real driving data using the method in [6], whereas the solid line is the transformed version that is equivalent to the New European Driving Cycle (NEDC) in propulsion requirement. This final transformation step is the topic of this paper, and a complete methodology is developed. It is based on a definition of equivalence for driving cycles that loosely speaking defines being similar without being the same. The result is a keystone in a design process for generation of driving cycles for better robustness in system design of vehicles, especially controls and diagnosis, with respect to real-world driving conditions. 


\section{A. Outline}

The outline of this paper is as follows. More background in the field is given in Section II. A characterization of the driving cycle is presented in Section III together with different measures related to the impact the driving cycle has on the vehicle. In Section IV a definition of driving cycle equivalence is given and in Section V the problem formulation is stated together with three main problems. A key contribution of this paper is the novel method for transforming driving cycles with general equivalence constraints in Section VI. Using the method the problems are solved in Section VII and finally in Section VIII the conclusions of this paper is presented.

\section{RELATED WORK}

Driving cycles have mainly been used to assess exhaust gas emissions of vehicles [3], [7]-[14], to evaluate different control strategies for vehicles [15]-[20], in vehicle design and sizing of components [21]-[27], and also in concept studies [2], [28]. A typical example of a legislative certification driving cycle for type approval for light-duty vehicles in Europe is the NEDC which is shown in Fig. 2. The reason for the fundamental role of driving cycles is that they give constraints that vehicles have to satisfy and where the cost, fuel consumption, and the pollutant emissions are all dependent on the driving cycle [29]-[35].

The certification driving cycles, such as the NEDC and the FTP75, are often used in comparison of vehicles. However, they are sometimes criticized to not be representative of realworld driving [25], [36], [37] since they are not as demanding compared to how vehicles are usually driven. To get a more demanding driving cycle one can either use a driving cycle generator approach or to change the given driving cycle so it becomes more demanding to follow. Examples of approaches that use the former case are different variants of the micro-tripapproach that is used in [3], [7], [8], [23], [38]-[40], the modebased approaches in [37], [41], and more recently variants of

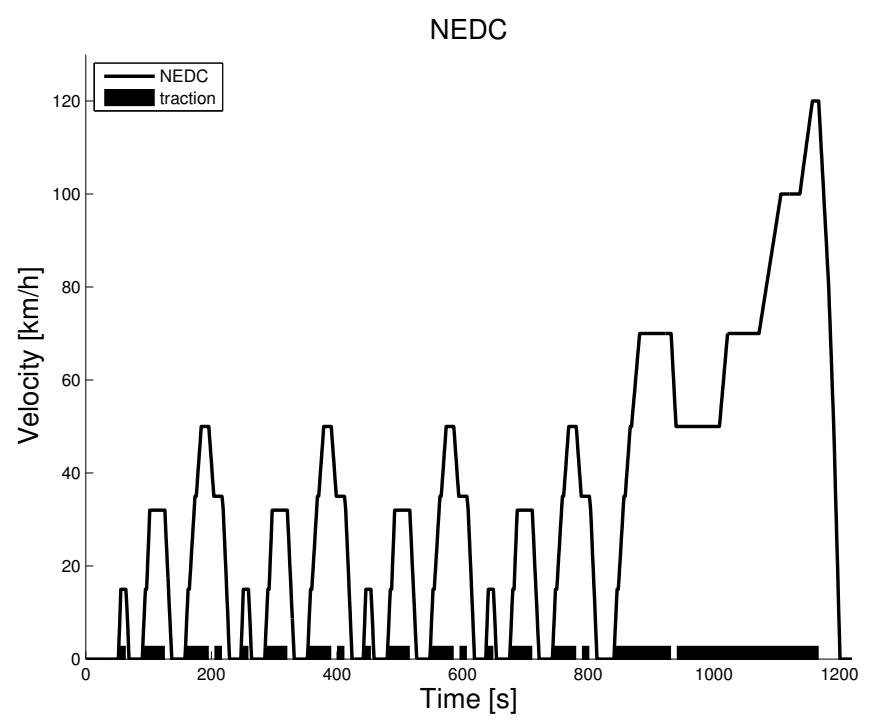

Fig. 2. NEDC with marked traction mode regions which indicates the instants where the powertrain needs to deliver positive power to the wheels. the Markov chain approach that use the vehicle speed and acceleration as states, see [6], [25], [42]-[44]. The latter case, to change the given driving cycle to a more demanding driving cycle is another option. In [45] a scaling of the UDDS driving cycle [46] was used. The vehicle speed in the UDDS was scaled by factors ranging from 1.1 to 1.4 and the time was inversely scaled by the same factors, resulting in an increase of acceleration by up to $96 \%$. Such a scaling results in that the mean speed and acceleration will increase while the driving distance will remain the same. However, other measures like the average power and maximum power are not as clear how much they will increase, resulting in a driving cycle that is more demanding but how much more demanding depends on both the driving cycle and the vehicle's parameters. Instead of scaling of the vehicle speed and the time in the driving cycle it would be beneficial to be able to insert different constraints on the driving cycle so the vehicle excitation of the driving cycle is better controlled during a change.

These questions were addressed in our previous work in [47] that proposed algorithms for transforming existing driving cycles while considering equivalence constraints on vehicle excitation. To be able to make a fair comparison of the results it is important that the vehicle has similar excitation for example regarding the forces acting on the vehicle, which lead to the fundamental concept of equivalence, similar but not the same, which was introduced in [47]. As equivalence measures mean tractive force, MTF, and its components were used. Those algorithms were somewhat limited since (i) only MTF components are considered, (ii) the changed driving cycles have some sharp peaks that reduced the driveability, and (iii) the algorithm could not change the vehicle speed in the nontraction regions in a systematic way. The work in this paper is a continuation of our previous work in [47] and here a new method is proposed for equivalence transformation of driving cycles. The contribution of this paper is a novel method that can transform a given driving cycle into a new driving cycle that (i) excites the vehicle in a similar way, (ii) considers driveability properties such that the driving cycle becomes easier to run in a chassis dynamometer, and (iii) fulfills imposed constraints, e.g., regarding the maximum acceleration, amount of recuperation energy, or maximum power. A key contribution of this work, compared to previously published works, is then the possibility to include such general constraints while keeping excitation levels such that experimental evaluations on the cycle can be compared to those on other driving cycles.

\section{Characterization OF Driving CyCles}

As mentioned previously the vehicle excitation is important if the comparison of the results in two or more driving cycles shall be fair. To be able to quantify how close two driving cycles are to each other regarding vehicle excitation, a measure is needed. Measures that previously have been used to characterize driving cycles are mostly related to different statistics of the driving cycle such as mean speed, standard deviation of acceleration, mean positive acceleration, and proportion of time spent in different vehicle modes such as acceleration, cruising, deceleration, and idling, see for example 
[33], [41], [48]. These measures reflect the driving cycle and not specifically the demand on the vehicle that has to follow the driving cycle.

In this paper measures that are based on the impact the driving cycle has on the vehicle will be used instead. The impact that a driving cycle has on a vehicle needs to be quantified, and for that a vehicle model is needed and will be presented in the Section III-A. In Section III-B the vehicle operating modes are defined by a partition of the driving cycle's timeline. Given the vehicle model these modes are determined by the required force at the wheels the powertrain needs to deliver for each given time.

A driving cycle yields demands that the vehicle has to fulfill. For example, the maximum acceleration and the maximum power need to be met by the powertrain if the vehicle shall be able to follow the driving cycle. Further, the power and energy demand in a driving cycle affects the sizing of components for all vehicles, and especially for electric and hybrid vehicles with an energy buffer where, e.g., the battery or electric machine need to be robustly sized. The measures used in this paper are the mean tractive force, MTF, components that are explained in Section III-C, and power and energy demands that are presented in Section III-D.

\section{A. Vehicle Model}

To be able to define measures on the driving cycle's demand on the vehicle, a vehicle model for the longitudinal dynamics is needed. The propulsion force, $F(t)$, at the wheels for flat roads consists of aerodynamic drag resistance, $F_{\text {air }}$, rolling friction resistance, $F_{\text {roll }}$, and inertia force, $F_{\mathrm{m}}$, for acceleration or deceleration of the vehicle. The three components are modeled as in [49]

$$
\begin{aligned}
F(t) & =F_{\text {air }}(t)+F_{\text {roll }}+F_{\mathrm{m}}(t) \\
F_{\text {air }}(t) & =\frac{1}{2} \rho_{a} c_{d} A_{f} v^{2}(t) \\
F_{\text {roll }} & =m g c_{r} \\
F_{\mathrm{m}}(t) & =m a(t),
\end{aligned}
$$

where $\rho_{a}$ is the air density, $c_{d}$ the drag coefficient, and the frontal area of the vehicle is denoted $A_{f}$. Further, the vehicle mass is $m, g$ is the gravitational constant, and the rolling friction coefficient is $c_{r}$. The vehicle speed is $v(t)$ and the acceleration of the vehicle is denoted $a(t)$.

\section{B. Vehicle Operating Modes}

A driving cycle consists of sequences of different vehicle modes, such as acceleration, cruising, deceleration, and idling mode. These vehicle modes are one possible partitioning of the whole time interval in a driving cycle $\tau=\left[t_{\text {start }}, t_{\text {end }}\right]$ where $t_{\text {start }}$ and $t_{\text {end }}$ are the start and final time in the driving cycle, respectively. The partitioning used in this paper is based on the excitation of the vehicle's powertrain instead of only based on vehicle speed or acceleration. The acceleration, cruising, and deceleration parts are here split into three vehicle operating modes denoted traction, coasting, and braking mode, see [49], which corresponds to the value of the vehicle propulsion force in (1). The three different vehicle operating modes, together with idling, are defined as

$$
\begin{aligned}
\tau_{\text {trac }} & =\{t \in \tau: F(t)>0, v(t) \neq 0\} \\
\tau_{\text {coast }} & =\{t \in \tau: F(t)=0, v(t) \neq 0\} \\
\tau_{\text {brake }} & =\{t \in \tau: F(t)<0, v(t) \neq 0\} \\
\tau_{\text {idle }} & =\{t \in \tau: v(t)=0\},
\end{aligned}
$$

where $\tau_{\text {trac }}$ is the traction mode region, $\tau_{\text {coast }}$ is the coasting mode region, $\tau_{\text {brake }}$ is when the vehicles brakes, and the idling set is denoted $\tau_{\text {idle }}$.

In Fig. 2 the NEDC, the legislative certification driving cycle for type approval for light-duty vehicles in Europe, is shown and the traction mode region is marked.

The partitioning in (5)-(8) will be used when introducing measures on the driving cycles, in the problem formulation in Section V, and as important parts in the method explained in Section VI. In the following sections, measures related to vehicle excitation will be presented.

\section{Mean Tractive Force Components}

The mean tractive force, MTF, is also sometimes called specific energy [42] or power intensity [50]. The MTF is described in [49], and is the vehicle's tractive energy at the wheels in a driving cycle, divided by distance traveled. It is a measure on how demanding the driving cycle is to follow for a given vehicle, and the higher MTF the higher demand on the vehicle.

A main idea in MTF is to consider the tractive force needed during a driving cycle by the following reasoning. Since the powertrain does not need to provide any positive forces to the wheels during coasting regions $(F(t)=0)$ or braking regions $(F(t)<0)$, the traction regions are those when the powertrain need to provide positive power to the wheels $(F(t)>0)$.

A driving cycle's MTF, $\bar{F}_{\text {trac }}$, is integrated over the set of intervals $\tau_{\text {trac }}$ as defined in (5) and is written as in [49]

$$
\bar{F}_{\text {trac }}=\frac{1}{x_{\text {tot }}} \int_{t \in \tau_{\text {trac }}} F(t) v(t) d t
$$

where $x_{\text {tot }}=\int_{t \in \tau} v(t) d t$ is the total distance traveled in the driving cycle. Thus, the power, $P(t)=F(t) v(t)$, at the wheels are integrated over the set $\tau_{\text {trac }}$ which yields the energy demand during the traction regions. The tractive energy is then divided by traveled distance and yields the MTF.

From (1) - (4) and (9) the MTF can be expressed by the sum of its components, denoted the MTF components, as

$$
\begin{aligned}
\bar{F}_{\text {trac }} & =\bar{F}_{\text {air }}+\bar{F}_{\text {roll }}+\bar{F}_{\mathrm{m}} \\
\bar{F}_{\text {air }} & =\frac{1}{x_{\text {tot }}} \int_{t \in \tau_{\text {trac }}} \frac{1}{2} \rho_{a} c_{d} A_{f} v^{3}(t) d t=\frac{1}{2} \rho_{a} c_{d} A_{f} \alpha(v(t)) \\
\bar{F}_{\text {roll }} & =\frac{1}{x_{\text {tot }}} \int_{t \in \tau_{\text {trac }}} m g c_{r} v(t) d t=m g c_{r} \beta(v(t)) \\
\bar{F}_{\mathrm{m}} & =\frac{1}{x_{\text {tot }}} \int_{t \in \tau_{\text {trac }}} m a(t) v(t) d t=m \gamma(v(t)),
\end{aligned}
$$


where the driving cycle characterizing measures $\alpha(v(t))$, $\beta(v(t))$, and $\gamma(v(t))$ are defined as

$$
\begin{aligned}
& \alpha(v(t))=\frac{\bar{F}_{\text {air }}}{\frac{1}{2} \rho_{a} c_{d} A_{f}}=\frac{1}{x_{\text {tot }}} \int_{t \in \tau_{\text {trac }}} v^{3}(t) d t \\
& \beta(v(t))=\frac{\bar{F}_{\text {roll }}}{m g c_{r}}=\frac{1}{x_{\text {tot }}} \int_{t \in \tau_{\text {trac }}} v(t) d t=\frac{x_{\text {trac }}}{x_{\text {tot }}} \\
& \gamma(v(t))=\frac{\bar{F}_{\mathrm{m}}}{m}=\frac{1}{x_{\text {tot }}} \int_{t \in \tau_{\text {trac }}} a(t) v(t) d t,
\end{aligned}
$$

where $x_{\text {trac }}$ is the distance traveled during the traction regions.

For a given vehicle the vehicle parameters are fixed. Two driving cycles, assuming the air density is constant, have then the same values on the MTF components, $\bar{F}_{\text {air }}, \bar{F}_{\text {roll }}$, and $\bar{F}_{\mathrm{m}}$, if they have the same values on $\alpha, \beta$, and $\gamma$ which are directly dependent on the driving cycle, and only affected indirectly by the vehicle parameters which affects $\tau_{\text {trac }}$. For further discussion see [47].

According to [49] the MTF can be used as an indication value of the fuel consumption. The relationship between the MTF components and the fuel consumption was investigated both in [44] with a simulation study and in [6] using a hardware-in-the-loop setup with a real engine. Both studies conclude that the MTF components better characterizes the fuel consumption compared to the aggregated MTF.

\section{Power and Energy Demand}

Hybrid electric vehicles, plug-in electric vehicles and also other types of hybrids do all have one or more degrees of freedom to choose which path the power will come from. This yields a possibility to reduce the fuel consumption by careful design of the power-split control. However, with the extra degree of freedom comes also sensitivity to the driving cycle, a poor design could lead to even worse fuel consumption. The reason for this is that the weight of the vehicle is usually increased, e.g., from an extra battery and electric machines, and if the control cannot be utilized well enough in the driving cycle the result can be even higher fuel consumption compared to conventional vehicles.

The power and energy demand in a driving cycle influences the design and the overall fuel efficiency of all vehicles and especially of hybrid and electric vehicles. The power and energy measures used in this paper are maximum power, average power, and amount of recuperative energy available. The average power in a driving cycle is here defined as

$$
P_{\text {avg }}=\frac{1}{\Delta t_{\text {tot }}} \int_{t \in \tau} F(t) v(t) d t
$$

where the total time in the driving cycle is $\Delta t_{\text {tot }}=t_{\text {end }}-t_{\text {start }}$.

The maximum power is

$$
P_{\max }=\max _{t} F(t) v(t) .
$$

The available recuperative energy in a driving cycle is equal to the amount of energy that is transformed to heat in the brake discs for a conventional vehicle during a driving cycle and is written as

$$
E_{\text {recup }}=-\int_{t \in \tau_{\text {brake }}} F(t) v(t) d t
$$

where the minus sign is to get positive $E_{\text {recup }}$ since during braking $F(t)<0$.

All these measures that have been introduced in this section will be used to illustrate the method that will be presented in Section VI.

\section{DRiving CyCle Equivalence}

To be able to determine if the vehicle excitation in two driving cycles are equivalent or not it is needed to define what equivalent driving cycles means. This is especially important if it is desired to do a fair comparison of the vehicle performance in different driving cycles, and in this paper it will also be used to show the performance of the method for the driving cycle transformation. Measures on how close a driving cycle is to another driving cycle or a complete driving cycle database are usually different statistics measures of the driving cycle. Examples are the aforementioned, mean speed, standard deviation of acceleration, and proportion of time in different vehicle modes. A driving cycle that is representative is a driving cycle which statistics are sufficiently close to normal driving, but no formal definition has been given.

In our previous work in [47] we denoted the term equivalent driving cycles that uses the characterizing measures (10)(12) and said that for a given vehicle, two driving cycles are equivalent with respect to the MTF components, or the characterizing measures, if these three measures are the same. Where, for a given vehicle means a set of model parameters, $\Omega=\left\{m, A_{f}, c_{d}, c_{v}, \rho_{a}, g\right\}$, used in the vehicle model in (2)(4). This paper will use an extension of the previous definition of equivalence. Let $\Gamma$ be a set of measures, $\theta_{i} \in \Gamma$, where $\theta_{i}$ are different measures, e.g., $\alpha$ in (10) or $P_{\text {avg }}$ in (13) and let $\Theta(v(t), \Omega)=\left(\theta_{1}(v(t), \Omega), \theta_{2}(v(t), \Omega), \ldots \theta_{n}(v(t), \Omega)\right)^{T}$. The extended definition is

Definition 1: For a given set of model parameters $\Omega$ and a set of measures $\Gamma$, two driving cycles, $v_{1}(t)$ and $v_{2}(t)$, are said to be equivalent with respect to the measures $\theta_{1}, \theta_{2}, \ldots, \theta_{n}$, denoted $v_{1}(t) \sim v_{2}(t)$, if

$$
\Theta\left(v_{1}(t), \Omega\right)=\Theta\left(v_{2}(t), \Omega\right) \text {. }
$$

Using $\Theta(v(t))=\left(\theta_{1}(v(t)), \theta_{2}(v(t)), \theta_{3}(v(t))\right)^{T}=$ $(\alpha(v(t)), \beta(v(t)), \gamma(v(t)))^{T}$, where the $\Omega$ dependence has been omitted, the extended definition coincides with the previous definition used in [6], [44], [47].

\section{Problem Formulation}

As previously discussed, a set of driving cycles are needed to avoid the risk of sub-optimal solutions based on a single driving cycle. This work approaches this problem by transforming a given driving cycle into a new, different, driving cycle but with similar properties such that the results can be compared. Therefore, transformation of a given driving cycle is a key problem in this work.

Before stating the fundamental problem, three motivating problem instances will be introduced that all will be put in a single framework and solved using the optimization based method described in Section VI. Below follows a brief introduction to the motivating problems, and then more detailed descriptions are given in Section V-B. 
The first motivating example, consider that there exist a driving cycle and also an additional test, e.g., an acceleration test from 70 to $120 \mathrm{~km} / \mathrm{h}$ that is now tested separately. To reduce the number of unique tests that needs to be performed, the acceleration test should be incorporated within the existing driving cycle. The problem is then, how shall the driving be changed so that it still excites the vehicle in a similar way as in the original driving cycle.

Second, in [8] it is proposed that low-powered vehicles shall be tested on other driving cycles than high-powered vehicles since the driving patterns differs due to the difference in engine performance. An alternative to create new driving cycles from new traffic measurements is to change an existing driving cycle to either a more demanding or less demanding driving cycle. Thus, an interesting problem is to start with a driving cycle and transform it to a new level of excitation, e.g., by changing the driving cycles MTF components.

Concept studies of hybrid or electric vehicles introduces additional possible constraints, for example requirements on power and recuperation regions. The third motivating problem thus introduces energy specific constraints, in addition to excitation constraint, on the driving cycle. As will be shown, the methodology is flexible and can introduce many types of constraints, for example driver comfort and driveability. Developing a general method that can handle a wide range of constraints is the objective in this paper.

\section{A. Transformable and Invariant Sets of the Driving Cycle}

When transforming a given driving cycle, $v$, it is desired to be able to control which regions that are allowed to change freely and which regions the user have direct control over. Such functionality will increase the usability of the method and to formalize this, the whole time-interval $\tau$ will be split into two sets as

$$
\tau=\tau_{\mathrm{t}} \cup \tau_{\text {inv }},
$$

where $\tau_{\mathrm{t}}$ and $\tau_{\text {inv }}$ are two disjoint sets. The set $\tau_{\mathrm{t}}$, denoted the transformable set, is where the method is allowed to change the vehicle speed while considering the constraints. The set $\tau_{\text {inv }}$ is the invariant set for which the vehicle speed in the driving cycle is determined directly be the user. The latter set can, e.g., contain a speed profile segment that shall remain the same during the transformation or if the user wants to incorporate a new speed profile, $v_{\text {spec }}$ at $\tau_{\text {spec }}$, within the existing driving cycle, which gives $\tau_{\text {spec }} \in \tau_{\text {inv }}$.

The traction and non-traction regions are not changed during the transformation of the driving cycle, that is $\tau_{\text {trac }}(\tilde{v})=$ $\tau_{\text {trac }}(v)$, where $\tilde{v}$ is the transformed driving cycle. However, the vehicle speed within each region can be changed. The transformable set is in all cases $\tau_{\mathrm{t}}=\tau \backslash \tau_{\text {spec }}$. Note that in [47] the transformable set was $\tau_{\mathrm{t}}=\tau \backslash\left(\tau_{\text {idle }} \cup \tau_{\text {coast }} \cup \tau_{\text {brake }} \cup \tau_{\text {spec }}\right)=$ $\tau_{\text {trac }} \backslash \tau_{\text {spec }}$. This means that the new method is more general compared to the previous method presented in [47].

The notation will be used in Section VII where the method will be used to find solutions for the problem instances that will be presented in the next section.

\section{B. Fundamental Problem and Three Problem Instances}

As motivated, the problem is to transform a given driving cycle into a new driving cycle, different from the first one, that fulfills a number of equivalence constraints. Thereby, performance of a design on the new driving cycle can be evaluated and compared to results on the original driving cycle since both driving cycles can be forced to have equivalent excitation properties. Generally, this can be formulated into the following problem.

Fundamental Problem: Based on a given driving cycle $v(t)$, a set of model parameters $\Omega$, and a set of target measures $\Theta^{\prime}=\left(\theta_{1}^{\prime}, \theta_{2}^{\prime}, \ldots \theta_{n}^{\prime}\right)^{T}$, the fundamental problem is to find a driving cycle $\tilde{v}(t)$ that fulfills $\Theta(\tilde{v}(t), \Omega)=\Theta^{\prime}$.

In Section V-A, three motivational examples were introduced which all can be formulated as particular instances P1, $\mathrm{P} 2$, and P3, of the fundamental problem above. Problems P1 and $\mathrm{P} 2$ are the same as in [47] while Problem P3 is new. For these three examples it will be shown how different problem settings all can be solved in a single framework using the method described in Section VI.

Problem P1 considers when a certain speed profile segment, $v_{\text {spec }}$ at $\tau_{\text {spec }}$, is to be incorporated in an existing drive cycle while the characterizing measures are kept constant during the transformation.

1) Problem P1: Given a driving cycle $v(t)$, a set of model parameters $\Omega$, and a set of target measures $\Theta^{\prime}=\Theta(v(t), \Omega)=$ $\left(\alpha^{\prime}, \beta^{\prime}, \gamma^{\prime}\right)^{T}$. Find $\tilde{v}(t) \neq v(t)$ so that (i) $\Theta(\tilde{v}(t), \Omega)=\Theta^{\prime}$ and (ii) $\tilde{v}(t)=v_{\text {spec }}(t), t \in \tau_{\text {spec }}$.

Next, in problem P2, the vehicle excitation of an existing driving cycle is changed by changing the characterizing measures.

2) Problem P2: Given a driving cycle $v(t)$, a set of model parameters $\Omega$, and a set of target measures $\Theta^{\prime}=\left(\alpha^{\prime}, \beta^{\prime}, \gamma^{\prime}\right)^{T}$. Find $\tilde{v}(t)$ so that $\Theta(\tilde{v}(t), \Omega)=\Theta^{\prime}$.

The target characterizing measures could be the values for another driving cycle $v_{2}(t)$, that is $\Theta^{\prime}=\Theta\left(v_{2}(t), \Omega\right)=$ $\left(\alpha^{\prime}, \beta^{\prime}, \gamma^{\prime}\right)^{T}$, and the resulting driving cycle, $\tilde{v}(t)$, would then be equivalent in those measures to $v_{2}(t)$, according to Definition 1 in Section IV.

A third problem, P3, considers in addition to the characterizing measures also constraints on vehicle power $P_{\text {avg }}$ and amount of energy in the recuperation regions $E_{\text {recup }}$.

3) Problem P3: Given a driving cycle $v(t)$, a set of model parameters $\Omega$, and a set of target measures $\Theta^{\prime}=$ $\left(\alpha^{\prime}, \beta^{\prime}, \gamma^{\prime}, P_{\text {avg }}, E_{\text {recup }}\right)^{T}$. Find $\tilde{v}(t)$ so that $\Theta(\tilde{v}(t), \Omega)=\Theta^{\prime}$.

\section{Method: Numerical Optimization APPROACH}

From the problem formulation the transformed driving cycle, $v=\left(v_{1}, v_{2}, \ldots, v_{n}\right)$, needs to fulfill a number of equality constraints that can be formulated as $h_{i}\left(v, \Omega, \Theta^{\prime}\right)=0$. Feasibility aspects such as that the driving cycle shall not cause the vehicle to exceed the maximum acceleration or that the vehicle operating regions shall be intact, can be formulated as inequality constraints as $g_{j}(v, \Omega) \leq 0$. Finding a method that can fulfill these constraints is sufficient with respect to 
the problem formulation, i.e., the problem is to solve

$$
\begin{aligned}
& h_{i}\left(v, \Omega, \Theta^{\prime}\right)=0 \\
& g_{j}(v, \Omega) \leq 0,
\end{aligned}
$$

with respect to $v$. The solution to (16)-(17) is typically not unique and there are several possibilities to find a feasible solution. One approach in our previous paper [47] was based on iterating the equations after a stochastic selection process and value modification followed by sorting. Another method, proposed now, is to also consider driveability of the resulting driving cycle. To do this, i.e., to also consider the smoothness of the driving cycle this paper introduces a cost function, $f(v)$, that allows minimizing of the vehicle jerk.

Using this, the transformation of the driving cycle can be formulated as a nonlinear program, NLP, with linear and nonlinear constraints and each optimization variable corresponds here to a specific vehicle speed in the driving cycle. The optimization can be formulated as

$$
\begin{aligned}
& \min f(v) \\
& \text { where } f: R^{n} \rightarrow R \\
& \text { s.t. } \\
& h_{i}(v)=0 \\
& g_{j}(v) \leq 0,
\end{aligned}
$$

where the dependence of the model parameters $\Omega$ and the target measures in $\Theta^{\prime}$ have been omitted.

The outline of this section is as follows. The constraints related to the vehicle operating modes in driving cycles, maximum acceleration constraints, and constraints regarding the characterizing measures will be formulated first. This is followed by the different power and energy constraints and constraints on the speed profile. Then the cost function that is minimized is formulated. Finally there is a summary and a discussion of the method.

\section{A. Constraints for Keeping the Traction and Non-Traction Regions}

The vehicle operating modes, which were introduced in Section III, are specified before the optimization starts. For example, if the vehicle shall remain in traction the vehicle speed cannot decrease too much from one vehicle speed point to next one in the driving cycle.

One way to characterize traction regions is to calculate the vehicle coasting speed, $v_{\text {coast }}$, which is determined by (1) and comparing it to the vehicle speed in the driving cycle. Using $\dot{v}(t)=a(t)$ and $F(t)=0$, the vehicle coasting speed is determined by

$$
\begin{aligned}
& 0=F_{\text {air }}+F_{\text {roll }}+F_{\mathrm{m}} \Rightarrow \\
& \dot{v}_{\text {coast }}(t)=-\frac{1}{2 m} \rho_{a} c_{d} A_{f} v_{\text {coast }}^{2}(t)-c_{r} g \\
&=-k_{1}^{2} v_{\text {coast }}^{2}(t)-k_{2}^{2} \Rightarrow \\
& v_{\text {coast }}\left(t, v\left(t_{k-1}\right)\right)=\frac{k_{2}}{k_{1}} \tan \left(\arctan \left(\frac{k_{1}}{k_{2}} v\left(t_{k-1}\right)\right)-\right. \\
&\left.k_{1} k_{2} \cdot\left(t-t_{k-1}\right)\right) .
\end{aligned}
$$

For driving cycles specified in discrete time and given an initial speed $v\left(t_{k-1}\right)$ in the driving cycle, the vehicle is in traction mode at time $t_{k}$ if $v\left(t_{k}\right)>v_{\text {coast }}\left(t_{k}, v\left(t_{k-1}\right)\right)$, otherwise the vehicle is in coasting mode if $v\left(t_{k}\right)=v_{\text {coast }}\left(t_{k}, v\left(t_{k-1}\right)\right)$, or in braking mode if $v\left(t_{k}\right)<v_{\text {coast }}\left(t_{k}, v\left(t_{k-1}\right)\right)$.

That is, if the traction regions, $\tau_{\text {trac }}$, in Section III, shall remain intact in the driving cycle the following constraints need to be fulfilled for all $t_{i} \in \tau_{\text {trac }}$

$$
\begin{aligned}
v_{\text {coast }}\left(t_{i}, v_{i-1}\right) & <v_{i} \Rightarrow \\
-v_{i}+v_{\text {coast }}\left(t_{i}, v_{i-1}\right) & <0,
\end{aligned}
$$

where $v_{i}=v\left(t_{i}\right)$. Each point in traction yields such an inequality as $\mathbf{g}_{1}(v)=\left(g_{1}(v), g_{2}(v), \ldots, g_{n_{\text {trac }}}(v)\right)<0$ where $n_{\text {trac }}$ is the number of vehicle speed points that are in traction.

Each point in the non-traction regions, $t_{j} \notin \tau_{\text {trac }}$, gives a corresponding inequality as

$$
\begin{aligned}
v_{j} & \leq v_{\text {coast }}\left(t_{j}, v_{j-1}\right) \Rightarrow \\
v_{j}-v_{\text {coast }}\left(t_{j}, v_{j-1}\right) & \leq 0,
\end{aligned}
$$

which leads to $n_{\text {non-trac }}$ number of inequality constraints where $n_{\text {non-trac }}$ is the number of speed points not in traction, $\mathbf{g}_{2}(v)=$ $\left(g_{n_{\text {trac }}+1}(v), g_{n_{\text {trac }}+2}(v), \ldots, g_{n_{\text {trac }}+n_{\text {non-trac }}}(v)\right) \leq 0$ where $n_{\text {trac }}+$ $n_{\text {non-trac }}=n$ is the number of speed points in the driving cycle.

\section{B. Constraints from Maximum Acceleration}

For a given vehicle, let $v_{i}$ and $a_{\max }\left(v_{i}\right)$ be the vehicle speed and its corresponding maximum acceleration for the vehicle at that speed, respectively. Since the powertrain can only deliver limited power to the wheels the next vehicle speed, $v_{i+1}$, in the driving cycle, cannot cause the vehicle to exceed the maximum acceleration. This is formulated as

$$
\begin{aligned}
\frac{v_{i+1}-v_{i}}{\Delta t} & \leq a_{\max }\left(v_{i}\right) \Rightarrow \\
v_{i+1}-\left(v_{i}+a_{\max }\left(v_{i}\right) \Delta t\right) & \leq 0,
\end{aligned}
$$

where $\Delta t$ is the sampling time of the driving cycle, that is the time between two speed points, and in this paper $\Delta t=1$.

This means that there are additional $n$ number of inequalities, one for each vehicle speed point, $\mathbf{g}_{3}(v)=$ $\left(g_{n+1}(v), g_{n+2}(v), \ldots, g_{2 n}(v)\right) \leq 0$.

The vehicle's maximum acceleration $a_{\max }\left(v_{i}\right)$ depends on the engine performance, on the vehicle parameters such as vehicle mass, and on the efficiency of the components. However, this paper uses a simplified expression of the maximum acceleration as used in [44] which reflects the maximum acceleration seen in normal driving as presented in [7, Fig.3]. The maximum acceleration used is

$$
a_{\max }\left(v_{\mathrm{km} / \mathrm{h}}\right)= \begin{cases}3 & \text { if } v \in(0,60] \\ 1.2-0.8 \cdot \frac{v_{\mathrm{km} h}-60}{100} & \text { if } v \in(60,160) .\end{cases}
$$

Note that the unit for vehicle speed is here expressed in $\mathrm{km} / \mathrm{h}$ and not in $\mathrm{m} / \mathrm{s}$ that is used in the other constraints. The unit for $a_{\max }$ is $\mathrm{m} / \mathrm{s}^{2}$.

Corresponding constraints regarding the maximum deceleration can also be used and it is trivial to add it if needed. However, no maximum deceleration constraints are used in this paper. 


\section{Constraints from Vehicle Excitation}

As described in Section III the MTF components are proportional to the driving cycle characterizing measures, $\alpha, \beta$, and $\gamma$ in (10) - (12), and are here always used as measures on vehicle excitation. Given target values on $\left(\alpha^{\prime}, \beta^{\prime}, \gamma^{\prime}\right)$, possibly from an existing driving cycle, the equality constraints for the vehicle excitation will be formulated in the following sections.

1) Constraint on $\alpha$ : Given a target value of $\alpha^{\prime}$, the driving cycle needs to fulfill the following equality in order to fulfill the constraint

$$
\begin{gathered}
\frac{1}{x_{t o t}} \int_{t \in \tau_{\text {trac }}} v(t)^{3} d t=\alpha^{\prime} \Rightarrow(\text { discrete }) \\
\frac{\sum_{i \in \tau_{\text {trac }}} v_{i}^{3} \Delta t}{\sum v_{i} \Delta t}-\alpha^{\prime}=0 \Rightarrow \\
h_{1}\left(v, \tau_{\text {trac }}, \alpha^{\prime}\right)=0,
\end{gathered}
$$

where $x_{\text {tot }}=\sum v_{i} \Delta t$ where $i \in \tau$. Further, $i \in \tau_{\text {trac }}$ are all indices where the corresponding vehicle speed point is in traction, see (19).

2) Constraint on $\beta$ : Given a target value of $\beta^{\prime}$, the driving cycle needs to fulfill

$$
\begin{gathered}
\frac{1}{x_{t o t}} \int_{t \in \tau_{\text {trac }}} v(t) d t=\beta^{\prime} \Rightarrow(\text { discrete }) \\
\frac{\sum_{i \in \tau_{\text {trac }}} v_{i} \Delta t}{\sum v_{i} \Delta t}-\beta^{\prime}=0 \Rightarrow \\
h_{2}\left(v, \tau_{\text {trac }}, \beta^{\prime}\right)=0 .
\end{gathered}
$$

3) Constraint on $\gamma$ : Given a target value of $\gamma^{\prime}$, the driving cycle needs to fulfill

$$
\begin{aligned}
& \frac{1}{x_{\text {tot }}} \int_{t \in \tau_{\text {trac }}} \dot{v}(t) \cdot v(t) d t=\gamma^{\prime} \Rightarrow \\
& \left.\frac{1}{x_{\text {tot }}} \int_{t \in \tau_{\text {trac }}} \frac{1}{2} \cdot \frac{d v^{2}(t)}{d t} d t-\gamma^{\prime}=0 \Rightarrow \text { (discrete }\right) \\
& \frac{\sum_{i}^{\# \operatorname{trac}}\left[\frac{v(t)^{2}}{2}\right]_{t_{i, \text { start }}}^{t_{i, \text { end }}}}{\sum v_{i} \Delta t}-\gamma^{\prime}=0 \Rightarrow \\
& h_{3}\left(v, \tau_{\text {trac }}, \gamma^{\prime}\right)=0,
\end{aligned}
$$

where \#trac is the number of traction regions, $t_{i, s t a r t}$ and $t_{i, e n d}$ are the start and end points in each traction region, respectively. The numerator is proportional to the difference in kinetic energy for all traction regions.

Note that the above mentioned equality constraints are calculated for the whole driving cycle and give three equality constraints, $h_{1}, h_{2}$, and $h_{3}$ corresponding to the characterizing measures of the driving cycle.

\section{Power and Energy Recuperation Constraints}

To be able to solve Problem P3 in Section V additional constraints considering power and energy recuperation need to be added. Two power constraints together with a constraint on the amount of recuperative energy in the driving cycle will now be formulated.
1) Power Constraints: The power in each time-point in a driving cycle can be calculated from the relationship $P(t)=$ $F(t) \cdot v(t)$ in (9). A constraint that the maximum power in (14) shall not exceed a certain maximum value, $P_{\max }^{\prime}$, is formulated as

$$
\begin{aligned}
\max _{t} F(t) \cdot v(t) & \leq P_{\max }^{\prime} \Rightarrow(\text { discrete }) \\
\max _{i} F\left(v_{i}\right) \cdot v_{i}-P_{\max }^{\prime} & \leq 0 \Rightarrow \\
g_{2 n+1}\left(v, \tau, P_{\max }^{\prime}\right) & \leq 0,
\end{aligned}
$$

where $F(t)=F(v(t))$.

Assume that the average power in (13) shall be a certain value $P_{\text {avg }}^{\prime}$. This yields an equality constraint as

$$
\begin{aligned}
\frac{1}{\Delta t_{\text {tot }}} \int_{t \in \tau} F(t) v(t) d t & =P_{\text {avg }}^{\prime} \Rightarrow(\text { discrete }) \\
\frac{\sum_{i} F\left(v_{i}\right) v_{i} \Delta t}{t_{\text {end }}-t_{\text {start }}}-P_{\text {avg }}^{\prime} & =0 \Rightarrow \\
h_{4}\left(v, \tau, P_{\text {avg }}^{\prime}\right) & =0 .
\end{aligned}
$$

2) Recuperation Energy Constraint: Assume that a driving cycle with target recuperative energy of $E_{\text {recup }}^{\prime}$, see (15), is wanted. This yields the following equality constraint

$$
\begin{aligned}
-\int_{t \in \tau_{\text {brake }}} F(t) v(t) d t & =E_{\text {recup }}^{\prime} \Rightarrow(\text { discrete }) \\
-\sum_{i \in \tau_{\text {brake }}} F\left(v_{i}\right) v_{i} \Delta t-E_{\text {recup }}^{\prime} & =0 \Rightarrow \\
h_{5}\left(v, \tau_{\text {brake }}, E_{\text {recup }}^{\prime}\right) & =0 .
\end{aligned}
$$

\section{E. Constraints on the Speed Profile}

Since the optimization variables are vehicle speed points in a driving cycle the nature of driving cycles limits the possible search space for the optimization variables. The lower bound is at least $v_{i} \geq 0$ and the upper bound can be set to the maximum speed, $v_{\max }$, of the vehicle, that is $v_{i} \leq v_{\max }$.

These bounds can be changed to solve certain problems. For example, assume that the idle set, $\tau_{\text {idle }}$, shall be the same before as after the transformation. To achieve this the lower and upper bound can be set to the same value as $l b\left(t \in \tau_{\text {idle }}\right)=$ $u b\left(t \in \tau_{\text {idle }}\right)=v\left(t \in \tau_{\text {idle }}\right)=0$ where $l b$ and $u b$ are the lower and upper bound, respectively, and where $v$ is the original driving cycle. This will ensure that the solver will output a driving cycle with the specified trajectory.

The same reasoning can be used to incorporate a certain speed profile segment, $v_{\text {spec }}$ at $\tau_{\text {spec }}$, as in Problem P1 in Section V. Using $l b\left(t \in \tau_{\text {spec }}\right)=u b\left(t \in \tau_{\text {spec }}\right)=v_{\text {spec }}\left(t \in \tau_{\text {spec }}\right)$. If a valid solution is found the requested speed profile will have been incorporated within the driving cycle.

\section{F. Cost Function}

As discussed below, there are many possible choices of cost function to minimize but in this paper the used cost function is

$$
f(v)=\|\ddot{v}\|_{2}^{2}
$$


Since $\ddot{v}=d^{2} v / d t^{2}$ is the jerk, the rate of change of acceleration, the optimization minimizes the jerk and thus gives a smooth driving cycle that increases the driveability. The cost function in (29) is used for all the problem instances in this general nonlinear optimization.

\section{G. Method Summary and Discussion}

Formulating the driving cycle transformation as an NLP is an effective way to include constraints, like on the vehicle speed, as well as measures on the whole driving cycle. The equality constraints $h_{i}$ in (18) can be used together with Definition 1 where two driving cycles are equivalent with respect to some measures if these measures are identical for the two driving cycles. The inequality constraints are more of the type that the driving cycle shall be feasible, e.g., by not exceeding the maximum acceleration as formulated in (22) or keeping the traction and non-traction regions as in (20) and (21). Regarding the cost function it should first be recalled that it is an alternative to the equation based approach in [47]. Further, that there are many possible alternatives for cost functions where one can think of variations that have more or less of a smoothing character. Here, the cost function used is the vehicle jerk which yields driving cycles that are smooth and thus have good driveability. Still, since the NLP formulation is general there are many more alternatives for cost functions to explore.

\section{RESULTS}

To illustrate the proposed methodology, the three problem instances that were introduced in Section $\mathrm{V}$ will be solved. Given a driving cycle, $v$, and a set of vehicle parameters according to Table I, the optimization formulation in (18) will be used with different constraints.

TABLE I

PARAMETERS FOR THE VEHICLE MODEL.

\begin{tabular}{lll}
\hline Parameter & Unit & Description \\
\hline$A_{f}=2.15$ & {$\left[\mathrm{~m}^{2}\right]$} & Frontal area \\
$c_{d}=0.4$ & {$[-]$} & Drag coefficient \\
$c_{r}=0.013$ & {$[-]$} & Rolling resistance coefficient \\
$m=1600$ & {$[\mathrm{~kg}]$} & Vehicle mass \\
$\rho_{a}=1.29$ & {$\left[\mathrm{~kg} / \mathrm{m}^{3}\right]$} & Air density \\
$g=9.81$ & {$\left[\mathrm{~m} / \mathrm{s}^{2}\right]$} & Gravitational constant \\
\hline
\end{tabular}

When solving Problem P1, the vehicle excitation regarding the MTF components shall remain the same while a specified speed profile for a certain sub-interval will be incorporated within the NEDC. When solving Problem P2, different driving cycles will have their vehicle excitation changed to another vehicle excitation with respect to the MTF components. The examples in this paper are to transform the NEDC and FTP75 to have the same vehicle excitation as each other. Additionally, a real-world driving cycle will also be transformed to be equivalent to NEDC. When solving Problem P3 additional constraints on the maximum power, average power and amount of energy for recuperation will be considered in the optimization. In that case the given driving cycle will be the NEDC and the output driving cycle will be a transformed variant of NEDC that fulfills the additional constraints.

For all problem instances presented here the traction and non-traction regions will be retained and for illustrative reasons the idling regions have remain unchanged during the transformations. The maximum acceleration will be considered by using $a_{\max }\left(v_{i}\right)$ as defined in Section VI-B. That is, the constraints (20) - (22) will be used in all the problem instances. The use of the other constraints, listed in (23)-(28) will vary from problem instance to problem instance.

The problems have been solved in Matlab with its function fmincon using an interior-point solver. The maximum functions evaluations was set to 30000 and each problem instances takes around 1-2 minutes to solve using a standard computer that has an Intel quad-core CPU of $2.66 \mathrm{GHz}$ and a memory of $3.7 \mathrm{~GB}$.

\section{A. Incorporating a Speed Profile - PI}

Given the NEDC, assume that a designer wants to incorporate a different speed profile segment starting at time $t=1072$. The segment, $v_{\text {spec }}$ at $\tau_{\text {spec }}=[1072,1088]$, to be incorporated within the NEDC, contains an acceleration from 70 to 120 $\mathrm{km} / \mathrm{h}$ which corresponds the normal maximum acceleration in Section VI-B. This will result in that the acceleration performance of the vehicle that follows the new driving cycle will be tested harder, but for reasons of comparison the excitation should be the same. In Fig. 3 the resulting driving cycle, $\tilde{v}$, from the optimization is shown as the solid line. The dashed line corresponds to NEDC and the difference in vehicle speed for the incorporated speed profile is clearly visible. These driving cycles are equivalent with respect to the characterizing measures. For vehicle parameters according to Table I the NEDC have the following characterizing measures $\left(\alpha_{\mathrm{NEDC}}, \beta_{\mathrm{NEDC}}, \gamma_{\mathrm{NEDC}}\right)=(317.1,0.86,0.11)$ and the equivalent version with the incorporated speed profile have a difference of $\left(1.3 \cdot 10^{-5}, 9.6 \cdot 10^{-5}, 0.02\right) \%$ in the characterizing measures. Thus, a negligible difference compared to the NEDC.

The total distance traveled, $x_{\text {tot }}$, is higher in $\tilde{v}$ but the two cycles are still equivalent with respect to the characterizing measures since the optimization raised the vehicle speed in the equivalent version of NEDC to compensate the change in $x_{\text {tot }}$.

1) Computational Complexity: The sampling time is one second and since the length of the NEDC is 1220 seconds and each vehicle speed in traction and non-traction is considered this results in 1220 different inequality constraints. Each speed point shall not exceed the maximum acceleration resulting in additional 1220 different inequality constraints. Three equality constraints were here used $\mathrm{h}_{1}, \mathrm{~h}_{2}$, and $\mathrm{h}_{3}$ from (23)-(25). The numbers of optimization variables are equal to the length of the driving cycle that is 1220 . However, 333 of these are in idle and additional 17 speed points originates from the incorporated speed profile. This results in that the number of optimization variables that the method can change freely, considering the constraints, are 870 in this case and there are 2400 inequality and 3 equality constraints to fulfill. 


\section{B. Transformation to Equivalent Driving Cycles - P2}

For illustrative purposes, the certification driving cycles, FTP75 and NEDC, will be transformed to a corresponding equivalent counterpart of each other, and thus be a solution for Problem P2. A more important use of P2 is as a component in data-driven driving cycle generation. Therefore, in addition, a generated real-world driving cycle from the driving cycle generator presented in [44] will be transformed to be equivalent to NEDC.

1) Transforming FTP75 to NEDC: Originally, for vehicle parameters according to Table I the FTP75 have the following characterizing measures $\left(\alpha_{\mathrm{FTP75}}, \beta_{\mathrm{FTP75}}, \gamma_{\mathrm{FTP75}}\right)=$ $(223.3,0.79,0.15)$. The corresponding values for NEDC and also the target values in the optimization are $\left(\alpha^{\prime}, \beta^{\prime}, \gamma^{\prime}\right)=$ $\left(\alpha_{\mathrm{NEDC}}, \beta_{\mathrm{NEDC}}, \gamma_{\mathrm{NEDC}}\right)=(317.1,0.86,0.11)$. This means that the constraints in (23) - (25), $h_{1}\left(v, \tau_{\text {trac }}, \alpha^{\prime}\right)=0$, $h_{2}\left(v, \tau_{\text {trac }}, \beta^{\prime}\right)=0$, and $h_{3}\left(v, \tau_{\text {trac }}, \gamma^{\prime}\right)=0$ will be used together with the constraints in (20) - (22).

The resulting driving cycle, $\tilde{v}$, from the optimization is shown in Fig. 4. The dashed line corresponds to the FTP75 and the solid line correspond to $\tilde{v}$ that is equivalent to NEDC, respectively. Since both $\alpha$ and $\beta$ need to be raised in the transformation the vehicle speed in the traction region need to be raised which can be seen by the increase in vehicle speed in the figure. Due to the transient behavior in the FTP75 the value of $\gamma$ is quite high and reflects that the driving cycle contains more accelerations followed by a coasting or braking region compared to the NEDC. The difference in the characterizing measures between the transformed FTP75 and NEDC are $\left(3.5 \cdot 10^{-5}, 2.8 \cdot 10^{-5}, 0.0054\right) \%$. The minimization of the cost function yields a minimization of the jerk in the driving cycle which is seen in the figure as the FTP75 has more changes in acceleration compared to the output from the optimization.

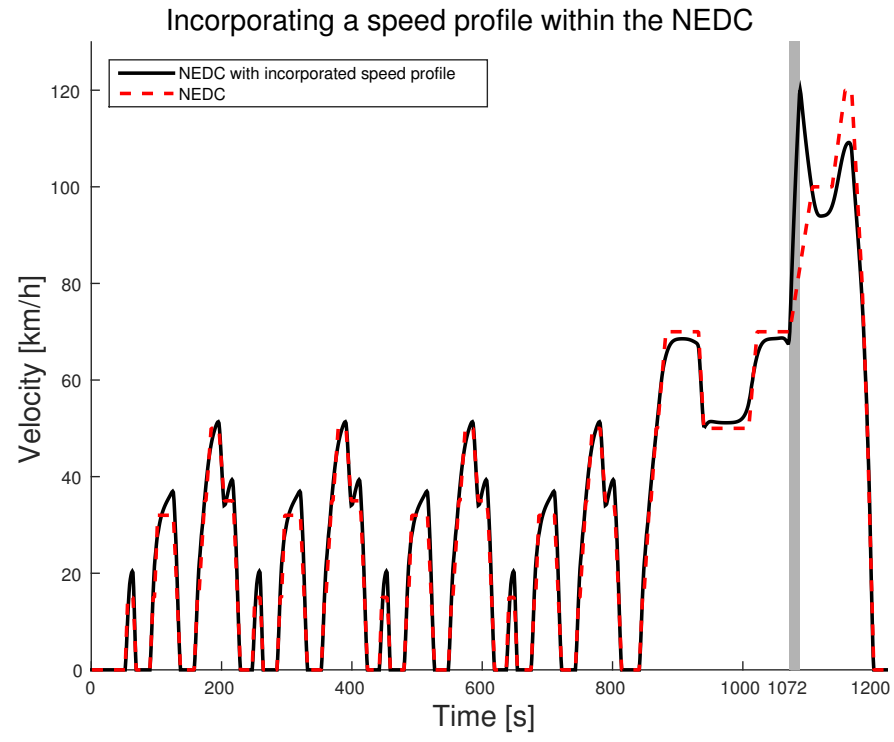

Fig. 3. NEDC (dashed) with an NEDC equivalent version (solid) with respect to the characterizing measures where a specified speed profile, $v_{\text {spec }}$, have been incorporated and is highlighted at $\tau_{\text {spec }}=[1072,1088]$.

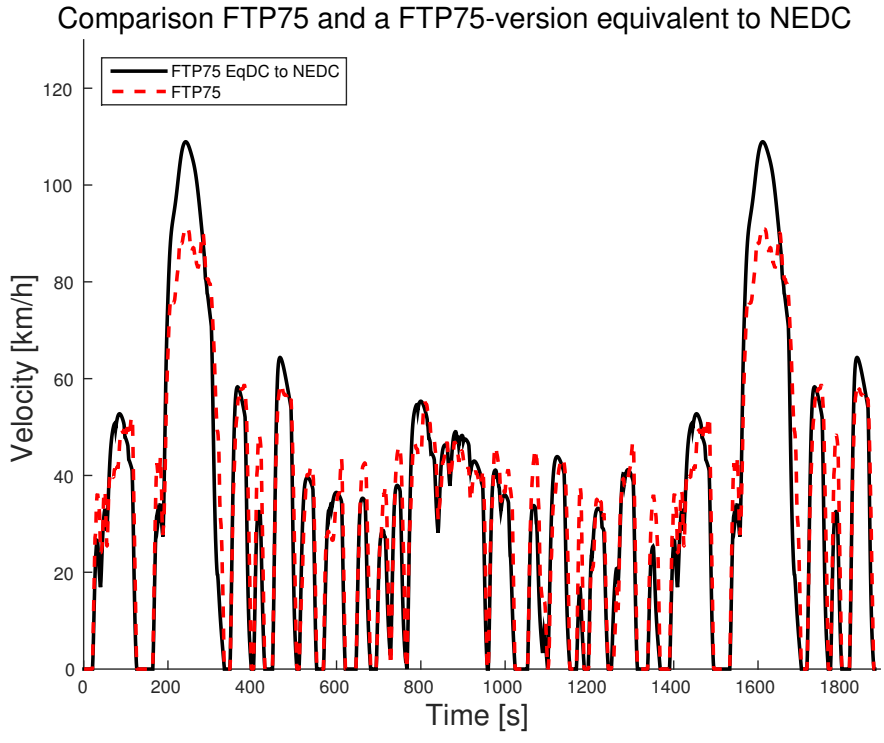

Fig. 4. FTP75 (dashed) and its equivalent version to NEDC (solid).

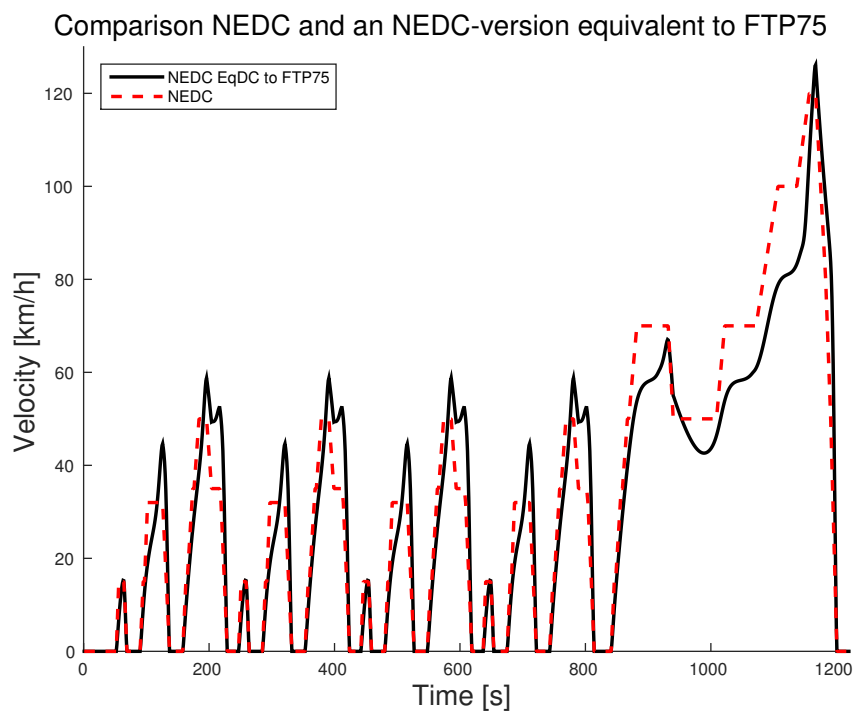

Fig. 5. NEDC (dashed) and its equivalent version to FTP75 (solid).

2) Transforming NEDC to FTP75: Here the NEDC will be transformed to be equivalent to FTP75. The resulting equivalent version is seen in Fig. 5 where the dashed line is the NEDC and the solid line is a driving cycle that is equivalent to FTP75.

Even if the mean speed in the FTP75 is higher than in the NEDC, (34.1 vs $33.6 \mathrm{~km} / \mathrm{h}$ ) the vehicle excitation constraints in (23) - (25) only consider vehicle speed in traction. To fulfill the constraints the vehicle speed need to be reduced during the traction regions, as can be seen have been done in the figure. The resulting driving cycle, $\tilde{v}$, have the following characterizing measures $(\alpha, \beta, \gamma)=(223.3,0.79,0.15)$ and the deviation compared to the target values are $\left(1.6 \cdot 10^{-4}, 1.3\right.$. $\left.10^{-4}, 2.8 \cdot 10^{-4}\right) \%$.

The minimization of the jerk is even more visible in this case. The driving cycle is smooth with fewer sharp edges compared to the NEDC. 
3) Real-World Driving Cycle: An important application of the method is to be used as a component in driving cycle generation tools where the generated driving cycle in addition to be representative to the database is desired to have equivalence properties. As an example, with the data-driven driving cycle generator in [44] a real-world driving database has been used to generate a driving cycle that is representative for the data in the database. In a next step it is desired for this driving cycle to be equivalent to NEDC, i.e. to have the same excitation as NEDC. The generated driving cycle has the following characterizing measures $(\alpha, \beta, \gamma)=(332.0,0.855,0.111)$. That driving cycle can be seen in Fig. 1 as the dashed line. Then the method has been applied and the resulting driving cycle, that is equivalent to NEDC, is the solid line in the figure. Compared to the generated driving cycle the equivalent driving cycle is much smoother and is equivalent to NEDC. The differences in the characterizing measures between the resulting driving cycle (solid line) in the figure and the NEDC are $\left(2.2 \cdot 10^{-4}, 0.01,0.009\right) \%$.

\section{Changing the Power and Energy in a Driving Cycle - P3}

When solving Problem P3, power and energy constraints also need to be considered. It will be illustrated by keeping the characterizing measures while the average power, maximum power, and amount of recuperative energy will be changed in the NEDC. The additional constraints used here are (23)-(28).

1) NEDC with Power and Recuperation Constraints: The amount of recuperate energy in NEDC for a vehicle with parameters according to Table $\mathrm{I}$ is $E_{\text {recup }}=1.33 \mathrm{MJ}$, the maximum power is $P_{\max }=41.8 \mathrm{~kW}$, and the average power is $P_{\text {avg }}=3.66 \mathrm{~kW}$.

Using the methodology the NEDC is transformed to a driving cycle that has $20 \%$ higher available recuperation energy, that is $E_{\text {recup }}^{\prime}=1.597 \mathrm{MJ}$ while the power constraints are set to $P_{\max }^{\prime}=40 \mathrm{~kW}$ and $P_{\mathrm{avg}}^{\prime}=4.0 \mathrm{~kW}$, respectively.

The resulting driving cycle, $\tilde{v}$, from the optimization is seen in Fig. 6. To get more available recuperation energy the vehicle needs to brake harder in the braking regions and increasing the average power causes a raise in the vehicle speed and thus the traveled distance. This has to be compensated by the method to fulfill the constraints regarding vehicle excitation in (23)-(25). The resulting driving cycle, $\tilde{v}$, have the following measures, $P_{\max }=38.81 \mathrm{~kW}, P_{\text {avg }}=4.000$ $\mathrm{kW}$, and $E_{\text {recup }}=1.597$ MJ. For the average power and amount of recuperative energy, which are equality constraints the deviation are $0.0027 \%$ and $0.009 \%$, respectively, which are negligible differences. The difference in the characterizing measures between the solid line in Fig. 6 and the NEDC (dashed line) are $\left(0.006,1.6 \cdot 10^{-6}, 0.05\right) \%$.

Summing up, non-trivial equivalence transformations are conveniently performed in one optimization problem.

\section{CONCLUSIONS}

Driving cycles play a fundamental role in vehicle design and development, especially in design and calibration of controls and diagnostics. To meet customer requirements who want their cars to perform well in real driving, requirements from

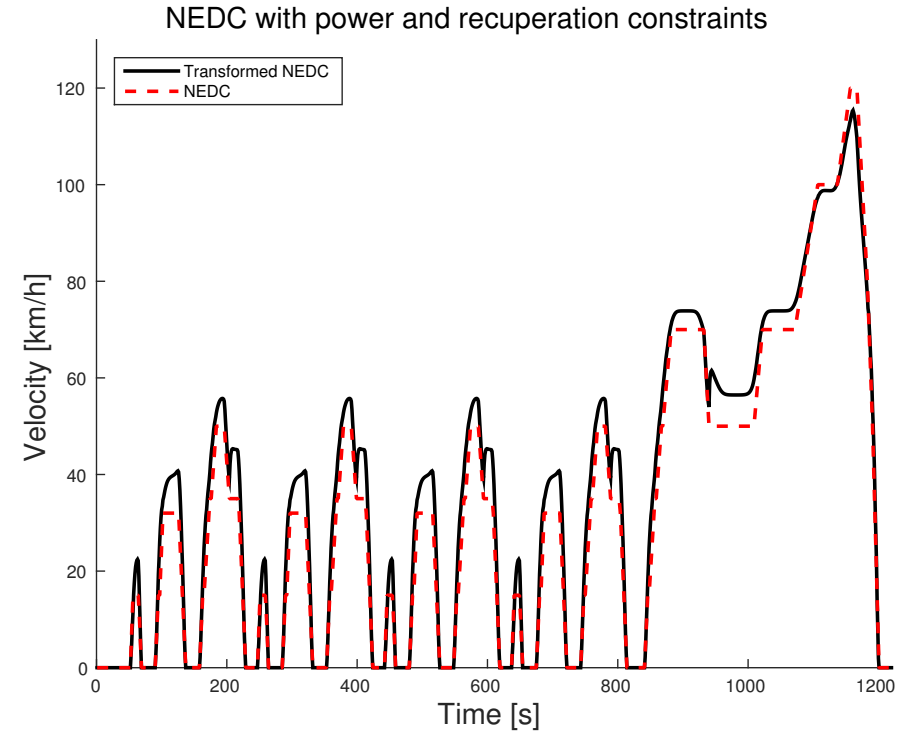

Fig. 6. NEDC (dashed) together with a transformed NEDC (solid) that have different measures regarding the amount of recuperation, maximum power and average power.

tests using PEMS (Portable Emission Measurement System), and requirements from upcoming new RDE (Real Driving Emissions) test procedures, design engineers must have easy and appropriate access to real driving behavior when designing, simulating, and testing their vehicle designs. Many methods are now being devised for utilizing driving databases, and to work well, they all need a final step that transforms a representative driving cycle obtained from the database into a driving cycle with prescribed traction requirements to be able to make fair comparative studies.

This final equivalence transformation is the topic of this paper, and we have presented a new, highly improved, and complete methodology to transform a given driving cycle to a new driving cycle that fulfills a number of equivalence constraints. The equivalence transformation of the driving cycle was formulated as a nonlinear program where the optimization variables are the vehicle speed points in the driving cycle. By using vehicle jerk as cost function the driveability of the driving cycle is simultaneously increased. An algorithm in Matlab was implemented, and three equivalence problems were solved to illustrate the transformation results. These and other similar transformations were solved in a few minutes. The method is general, easy to use, and can include high-level constraints such as the desired amount of energy in recuperation regions $E_{\text {recup }}$, and also other types of the constraints not illustrated in this paper. Further, the level of modification can be controlled and directed to parts of the driving cycle of specific interest.

In conclusion, for a wide set of useful equivalence formulations for driving cycles, approaching the important problem of similar but not the same, the nonlinear program with jerk as cost function effectively gives solutions to real-world problems. The method is straightforward to use as a component in data-driven methods for finding representative driving cycles with equivalence properties, and all in all it gives an effective engineering tool for all these problems. 


\section{REFERENCES}

[1] K. Nesamani and K. Subramanian, "Impact of real-world driving characteristics on vehicular emissions," JSME International Journal, Series B: Fluids and Thermal Engineering, vol. 49, no. 1, pp. 19-26, 2006.

[2] S. Onoda and A. Emadi, "PSIM-based modeling of automotive power systems: Conventional, electric, and hybrid electric vehicles," Vehicular Technology, IEEE Transactions on, vol. 53, no. 2, pp. 390-400, March 2004.

[3] Q. Wang, H. Huo, K. He, Z. Yao, and Q. Zhang, "Characterization of vehicle driving patterns and development of driving cycles in Chinese cities," Transportation Research Part D: Transport and Environment, vol. 13, no. 5, pp. $289-297,2008$.

[4] E. Tazelaar, J. Bruinsma, B. Veenhuizen, and P. van den Bosch, "Driving cycle characterization and generation, for design and control of fuel cell buses," World Electric Vehicle Journal, vol. 3, no. 1, 2009.

[5] V. Schwarzer and R. Ghorbani, "Drive cycle generation for design optimization of electric vehicles," IEEE Transactions on Vehicular Technology, vol. 62, no. 1, pp. $89-97,2013$.

[6] P. Nyberg, E. Frisk, and L. Nielsen, "Using real-world driving databases to generate driving cycles with equivalence properties," Vehicular Technology, IEEE Transactions on, vol. Accepted, In Early Access, no. 99, 2016.

[7] M. André, "The ARTEMIS European driving cycles for measuring car pollutant emissions," Science of The Total Environment, vol. 334-335, no. 0 , pp. $73-84,2004$.

[8] M. André, R. Joumard, R. Vidon, P. Tassel, and P. Perret, "Real-world European driving cycles, for measuring pollutant emissions from highand low-powered cars," Atmospheric Environment, vol. 40, no. 31, pp. $5944-5953,2006$.

[9] W. Wang, D. Lyons, N. Clark, M. Gautam, and P. Norton, "Emissions from nine heavy trucks fueled by diesel and biodiesel blend without engine modification," Environmental Science and Technology, vol. 34, no. 6, pp. 933-939, 2000.

[10] G. Fontaras, G. Karavalakis, M. Kousoulidou, T. Tzamkiozis, L. Ntziachristos, E. Bakeas, S. Stournas, and Z. Samaras, "Effects of biodiesel on passenger car fuel consumption, regulated and non-regulated pollutant emissions over legislated and real-world driving cycles," Fuel, vol. 88, no. 9, pp. $1608-1617,2009$.

[11] L. Fu, J. Hao, D. He, K. He, and P. Li, "Assessment of vehicular pollution in China," Journal of the Air and Waste Management Association, vol. 51, no. 5, pp. 658-668, 2001.

[12] C.-C. Chan, C.-K. Nien, C.-Y. Tsai, and G.-R. Her, "Comparison of tailpipe emissions from motorcycles and passenger cars," Journal of the Air and Waste Management Association, vol. 45, no. 2, pp. 116-124, 1995.

[13] E. Zervas and G. Bikas, "Impact of the driving cycle on the NOx and particulate matter exhaust emissions of diesel passenger cars," Energy and Fuels, vol. 22, no. 3, pp. 1707-1713, 2008.

[14] L. Pelkmans and P. Debal, "Comparison of on-road emissions with emissions measured on chassis dynamometer test cycles," Transportation Research Part D: Transport and Environment, vol. 11, no. 4, pp. $233-$ 241, 2006.

[15] P. Pisu and G. Rizzoni, "A comparative study of supervisory control strategies for hybrid electric vehicles," Control Systems Technology, IEEE Transactions on, vol. 15, no. 3, pp. 506-518, May 2007.

[16] M. Koot, J. Kessels, B. de Jager, W. Heemels, P. van den Bosch, and M. Steinbuch, "Energy management strategies for vehicular electric power systems," Vehicular Technology, IEEE Transactions on, vol. 54, no. 3, pp. 771-782, May 2005.

[17] C. Manzie, H. Watson, and S. Halgamuge, "Fuel economy improvements for urban driving: Hybrid vs. intelligent vehicles," Transportation Research Part C: Emerging Technologies, vol. 15, no. 1, pp. 1-16, 2007.

[18] J. Park, Z. Chen, L. Kiliaris, M. Kuang, M. Masrur, A. Phillips, and Y. Murphey, "Intelligent vehicle power control based on machine learning of optimal control parameters and prediction of road type and traffic congestion," IEEE Transactions on Vehicular Technology, vol. 58, no. 9, pp. 4741-4756, 2009.

[19] J.-P. Gao, G.-M. Zhu, E. Strangas, and F.-C. Sun, "Equivalent fuel consumption optimal control of a series hybrid electric vehicle," Proceedings of the Institution of Mechanical Engineers, Part D: Journal of Automobile Engineering, vol. 223, no. 8, pp. 1003-1018, 2009.

[20] S. Stockar, V. Marano, M. Canova, G. Rizzoni, and L. Guzzella, "Energy-optimal control of plug-in hybrid electric vehicles for realworld driving cycles," IEEE Transactions on Vehicular Technology, vol. 60 , no. 7 , pp. $2949-2962,2011$
[21] C. N. Maxoulis, D. N. Tsinoglou, and G. C. Koltsakis, "Modeling of automotive fuel cell operation in driving cycles," Energy Conversion and Management, vol. 45, no. 4, pp. 559 - 573, 2004.

[22] J. Hellgren and E. Jonasson, "Maximisation of brake energy regeneration in a hybrid electric parallel car," International Journal of Electric and Hybrid Vehicles, vol. 1, no. 1, pp. 95-121, 2007.

[23] R. Smith, S. Shahidinejad, D. Blair, and E. Bibeau, "Characterization of urban commuter driving profiles to optimize battery size in light-duty plug-in electric vehicles," Transportation Research Part D: Transport and Environment, vol. 16, no. 3, pp. 218 - 224, 2011.

[24] N. Murgovski, L. Johannesson, J. Sjöberg, and B. Egardt, "Component sizing of a plug-in hybrid electric powertrain via convex optimization," Mechatronics, vol. 22, no. 1, pp. 106-120, 2012.

[25] G. Souffran, L. Miegeville, and P. Guerin, "Simulation of real-world vehicle missions using a stochastic markov model for optimal powertrain sizing," IEEE Transactions on Vehicular Technology, vol. 61, no. 8, pp. $3454-3465,2012$

[26] A. Jaafar, B. Sareni, and X. Roboam, "A systemic approach integrating driving cycles for the design of hybrid locomotives," IEEE Transaction on Vehicular Technology, vol. 62, no. 8, pp. 3541-3550, 2013.

[27] M. Pourabdollah, N. Murgovski, A. Grauers, and B. Egardt, "Optimal sizing of a parallel PHEV powertrain," IEEE Transactions on Vehicular Technology, vol. 62, no. 6, pp. 2469-2480, 2013.

[28] R. Wolfson and J. Gower, "The role of computer modeling and simulation in electric and hybrid vehicle research and development," Vehicular Technology, IEEE Transactions on, vol. 32, no. 1, pp. 62-73, Feb 1983.

[29] B. Liaw and M. Dubarry, "From driving cycle analysis to understanding battery performance in real-life electric hybrid vehicle operation," Journal of Power Sources, vol. 174, no. 1, pp. 76 - 88, 2007.

[30] T. D. Durbin, R. D. Wilson, J. M. Norbeck, J. Miller, T. Huai, and S. H. Rhee, "Estimates of the emission rates of ammonia from light-duty vehicles using standard chassis dynamometer test cycles," Atmospheric Environment, vol. 36, no. 9, pp. 1475 - 1482, 2002.

[31] A. Kean, R. Harley, and G. Kendall, "Effects of vehicle speed and engine load on motor vehicle emissions," Environmental Science and Technology, vol. 37, no. 17, pp. 3739-3746, 2003.

[32] M. André and M. Rapone, "Analysis and modelling of the pollutant emissions from European cars regarding the driving characteristics and test cycles," Atmospheric Environment, vol. 43, no. 5, pp. 986 - 995, 2009.

[33] E. Ericsson, "Variability in urban driving patterns," Transportation Research Part D: Transport and Environment, vol. 5, no. 5, pp. 337 $-354,2000$.

[34] — "Independent driving pattern factors and their influence on fueluse and exhaust emission factors," Transportation Research Part D: Transport and Environment, vol. 6, no. 5, pp. 325 - 345, 2001.

[35] A. Kristensson, C. Johansson, R. Westerholm, E. Swietlicki, L. Gidhagen, U. Wideqvist, and V. Vesely, "Real-world traffic emission factors of gases and particles measured in a road tunnel in Stockholm, Sweden," Atmospheric Environment, vol. 38, no. 5, pp. 657 - 673, 2004.

[36] J.-M. Zaccardi and F. Le Berr, "Analysis and choice of representative drive cycles for light duty vehicles - Case study for electric vehicles," Proceedings of the Institution of Mechanical Engineers, Part D: Journal of Automobile Engineering, vol. 227, no. 4, pp. 605-616, 2013.

[37] A. Ashtari, E. Bibeau, and S. Shahidinejad, "Using large driving record samples and a stochastic approach for real-world driving cycle construction: Winnipeg driving cycle," Transportation Science, vol. 48, no. 2, pp. 170-183, 2014

[38] J. Kent, G. Allen, and G. Rule, "A driving cycle for Sydney," Transportation Research, vol. 12, no. 3, pp. 147 - 152, 1978.

[39] H. Tong, W. Hung, and C. Cheung, "Development of a driving cycle for Hong Kong," Atmospheric Environment, vol. 33, no. 15, pp. 2323 2335, 1999.

[40] S. Shahidinejad, E. Bibeau, and S. Filizadeh, "Statistical development of a duty cycle for plug-in vehicles in a North American urban setting using fleet information," IEEE Transactions on Vehicular Technology, vol. 59, no. 8, pp. 3710-3719, 2010.

[41] J. Lin and D. Niemeier, "An exploratory analysis comparing a stochastic driving cycle to Californias regulatory cycle," Atmospheric Environment, vol. 36, no. 38, pp. 5759-5770, 2002.

[42] T.-K. Lee and Z. Filipi, "Synthesis of real-world driving cycles using stochastic process and statistical methodology," International Journal of Vehicle Design, vol. 57, no. 1, pp. 17-36, 2011.

[43] Q. Gong, S. Midlam-Mohler, V. Marano, and G. Rizzoni, "An iterative markov chain approach for generating vehicle driving cycles," $S A E$ International Journal of Engines, vol. 4, no. 1, pp. 1035 - 1045, 2011. 
[44] P. Nyberg, E. Frisk, and L. Nielsen, "Generation of equivalent driving cycles using markov chains and mean tractive force components," in 19th IFAC World Congress, vol. 19, no. 1, Cape Town, South Africa, 2014, pp. 8787-8792.

[45] R. Carlson, H. Lohse-Busch, M. Douba, and N. Shidore, "Drive cycle fuel consumption variability of plug-in hybrid electric vehicles due to aggressive driving," in SAE Technical paper 2009-01-1335, Detroit, USA, 2009.

[46] R. E. Kruse and T. A. Huls, "Development of the federal urban driving schedule." SAE Prepr, no. 730553, 1973.

[47] P. Nyberg, E. Frisk, and L. Nielsen, "Driving cycle adaption and design based on mean tractive force," in 7th IFAC Symposium on Advances in Automotive Control, vol. 7, no. 1, Tokyo, Japan, 2013, pp. 689 - 694.

[48] M. André, "Driving cycles development: Characterization of the methods," in SAE Technical Paper 961112, 1996.

[49] L. Guzzella and A. Sciarretta, Vehicle Propulsion System: Introduction to Modeling and Optimization. Springer, 2007.

[50] E. Tate, M. Harpster, and P. Savagian, "The electrification of the automobile: From conventional hybrid, to plug-in hybrids, to extendedrange electric vehicles," SAE International Journal of Passenger Cars Electronic and Electrical Systems, vol. 1, no. 1, pp. 156-166, 2008.

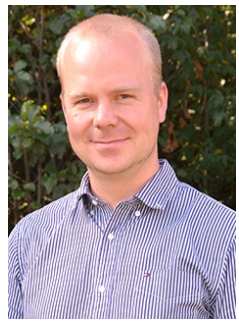

Peter Nyberg was born in Sweden in 1983. He received the M.Sc. degree in applied physics and electrical engineering and the Ph.D. degree in vehicular systems from Linköping University, Linköping, Sweden, in 2009 and 2015, respectively. His research interests include vehicle testing, optimization, and driving cycles.

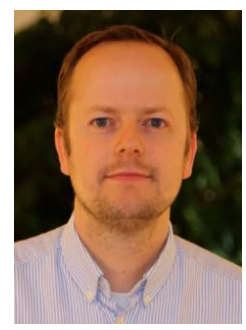

Erik Frisk was born in Sweden in 1971. He received the Ph.D. degree in 2001 from Linköping University, Linköping, Sweden. Currently he has a position as an Associate Professor at the Department of Electrical Engineering, Linköping University, Sweden. His research interests include issues in model based fault diagnosis and fault isolation, prognostics, and observer theory.

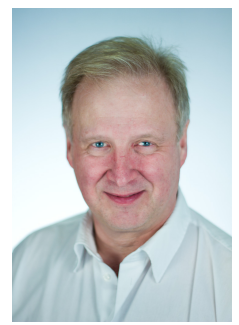

Lars Nielsen was born in Sweden in 1955 . He received the M.Sc. degree in engineering physics and the $\mathrm{Ph} . \mathrm{D}$. degree in automatic control from Lund Institute of Technology, Lund, Sweden, in 1979 and 1985, respectively. Since 1992, he has been a Professor with the Division of Vehicular Systems, Department of Electrical Engineering, Linköping University, Linköping, Sweden, where he holds the Sten Gustafsson Chair. His research interests include automotive modeling, control, diagnosis, and autonomous functions. 